\title{
FLUID-THERMAL CO-SIMULATION FOR A HIGH PERFORMANCE CONCRETE MACHINE FRAME
}

\author{
Christoph Steiert $^{1 *}$, Juliane Weber ${ }^{1}$, Alexander Galant $^{2}$, Janine Glänzel ${ }^{3}$, Jürgen Weber ${ }^{1}$ \\ 1 Institute of Mechatronic Engineering, Chair of Fluid-Mechatronic Systems, Technische Universität Dresden, \\ Helmholtzstrasse 7a, 01069 Dresden \\ 2 Institute of Mechatronic Engineering, Chair of Machine Tools Development and Adaptive Controls, Technische \\ Universität Dresden \\ 3 Fraunhofer Institute for Machine Tools and Forming Technology IWU \\ * Corresponding author: Tel.: +49 351 46333989; E-mail address: christoph.steiert@tu-dresden.de
}

\begin{abstract}
Thermo-elastic errors are one of the main drivers for reduced quality of workpieces in machining. Cooling systems can prevent these errors and improve quality. The paper describes a simulation method that takes into account both the temperature field of a machine tool frame and the fluid cooling system. Such simulations can help to improve the thermal stability of the machine tool frame.
\end{abstract}

Keywords: thermal simulation, model order reduction, cooling system, modeling

\section{INTRODUCTION}

The thermo-energetic behaviour of machine tools has recently become more and more important. A main driver for this development is to improve the workpiece quality and the energy efficiency of the machine. Power losses that occur during production induce heat in the machine tool and lead to temperature gradients within the structure. These temperature gradients are one reason for a tool centre point (TCP) displacement, which leads to a loss of quality in manufacturing. For this reason, power losses should be reduced and, if this is not affordable, the thermal state of the machine tool has to be stabilised. Fluidic cooling systems offer a good mechanism to do this by transporting heat from the critical workspace.

This paper presents a simulation method for investigating the thermal behaviour of a machine tool frame. The simulation consists of two parts. First, a fast calculating thermal finite element method (FEM) simulation of the machine tool frame and second, a network-based simulation of the fluid cooling system.

\section{STATE OF THE ART}

In recent years there were projects investigating the thermo-energetic behavior of machine tools [1-3]. The improvement of quality and productivity of machine tools and the reduction of energy consumption motivated these projects. For example, $75 \%$ of the overall geometrical errors of workpieces are induced by the effects of varying temperatures in the machine tool [4]. Cooling mechanisms should transport heat from the machine tools. For this purpose, water or water-glycol mixtures are often used as cooling medium, as these have a higher relative heat capacity in comparison to air. The efficiency of fluid system components such as compressors, pumps, electric drives improved in the past as well as control strategies. Now the cooling unit often works in switching mode, so that no energy is needed in idle phases, but it was shown in [5] that this behavior, together with the use of constant drive pumps, has a negative influence on the temperature field in the machine tool. For better thermal stability in connection with an energetically better behavior, it is recommended to use variable speed drive pumps or control valves [6]. With these tools a demand-oriented cooling strategy is feasible.

Simulations are able to predict the thermal behavior of machine tools. For such simulations FEM is chosen. The models are quite complex and need computational effort if high accuracy is required. For analysis and controller design a long computing time is not acceptable. A solution for this problem are model order reduction 
(MOR) methods based on Krylov subspace methods [7]. This method is used successfully in [8].

The research presented in this paper takes up the aspects described and applies them to the research object machine tool frame. In the view of the authors, the implementation of a coupled finite element (FE) model of a machine frame with a network-based model of a fluid system delivers new aspects to improve the thermal behavior of machine tool frames.

\section{TEST RIG}

The test rig consists of two subsystems: the solid machine tool frame and the fluidic cooling system. Hoses connect both subsystems.

\subsection{Machine tool frame}

The machine tool frame has a base area of $3.0 \mathrm{x}$ $4.0 \mathrm{~m}$ and a height of $1.6 \mathrm{~m}$. It is made of a high performance concrete (HPC) for better thermal properties and has a weight of $18.0 \mathrm{t}$. On top of the U-shaped machine frame, a parallel kinematic machine tool can be placed. The research presented in this paper is done without this parallel kinematic in order to focus specifically on the tempering of the HPC machine tool frame by means of the integrated six cooling channels. Their piping is made from steel. The cooling channels are located where the highest heat input from the process and the periphery is assumed. In Figure 1 these locations are shown. They are under the linear guiding system on each side F1 and F2, the chip collectors S1 and S2 and in the mounting flanges of the two drives M1 and M2. The measuring of the temperature field inside of the HPC machine tool frame occurs with the help of 23 temperature sensors. Figure 2 shows the position of the sensors and their labels. The sensors are mapped to different regions of the machine tool frame, according to Table 1.

Beside the temperature sensors inside the machine tool frame one has also temperature sensors on the edge of the machine tool frame and in the environment to get the temperature in different heights.

\subsection{Fluidic tempering system}

For tempering the machine tool frame a fluidic system is used, which consists of actors, sensors and connecting elements.
As actuators suit a fluid-air cooling system (FLKS), valves and a pool heater. The elements of the FLKS are a tank, a pump and a fluid-air heat exchanger. The tank is a reservoir for the cooling medium with a capacity of $31.0-41.01$.

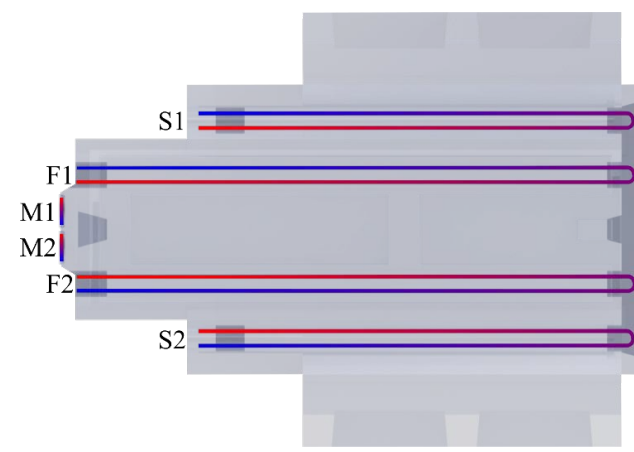

Figure 1: Machine tool frame with cooling channels, top view
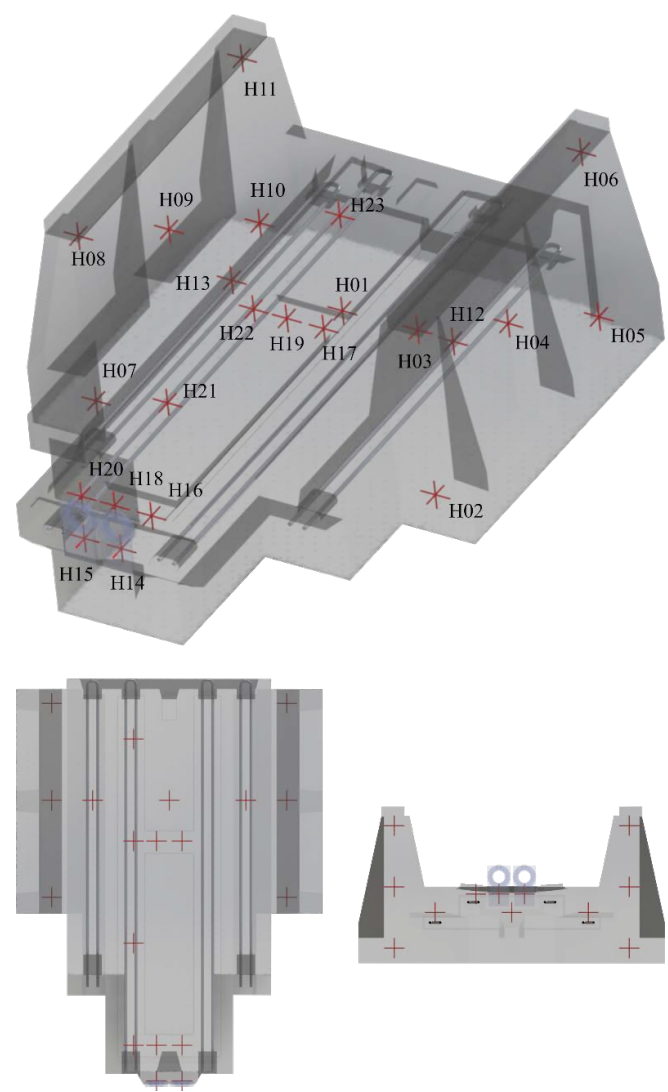

Figure 2: Machine frame with temperature sensors 
Table 1: Sensor mapping

\begin{tabular}{ll}
\hline Region & Sensors \\
\hline core & $\mathrm{H} 01, \mathrm{H} 18, \mathrm{H} 19$ \\
left side & $\mathrm{H} 02-\mathrm{H} 06$ \\
right side & $\mathrm{H} 07-\mathrm{H} 11$ \\
left chip collector & $\mathrm{H} 12$ \\
right chip collector & $\mathrm{H} 13$ \\
left drive & $\mathrm{H} 14$ \\
right drive & $\mathrm{H} 15$ \\
left linear guidance & $\mathrm{H} 16, \mathrm{H} 17$ \\
right linear guidance & $\mathrm{H} 20-\mathrm{H} 23$ \\
\hline
\end{tabular}

The pump is a centrifugal pump that delivers a volume flow rate $\dot{V}$ of $55.0 \mathrm{l} / \mathrm{min}$ at 2.8 bar. A constant speed drive unit powers the pump, so that there is no possibility to manipulate the amount of volume flow with the pump. The last component of the FLKS is the fluid/air heat exchanger with an integrated temperature control. With this control, one has the possibility to set the fan speed and to maintain a constant fluid temperature or the fluid temperature follows the ambient temperature with a desired offset $\Delta T$ of e. g. $5.0 \mathrm{~K}$. In the use cases presented in section 5 , the fan is not active.

The distribution of the correct amount of volume flow $\dot{V}$ is the task of six $3 / 2$ proportional directional control valves. The valves themselves are controlled by a $0.0 \ldots 10.0 \mathrm{~V}$ signal. A PIcontroller sets the input signal for each valve. The volume flow not required is returned to the tank via a bypass pipe.

For tempering the volume flow a $3.0 \mathrm{~kW}$ pool heater is used. The pool heater enables the user to set a desired temperature. It operates as a switching two-point control with a peak-to-peak value of $3.0 \mathrm{~K}$. The used cooling medium is water. The test cycles presented in section 5 are designed to heat the machine tool frame and not to cool it. This is done to bring more heat in the machine frame, by having larger temperature gradients between the fluid and the solid of the machine frame. This inverse procedure is sufficient in particular for the validation of the simulation model (from section 4) with regard to the temporal and local temperature behavior. In addition, the uncertainties when installing external heat sources and determining their heat input into the frame structure can be reduced.

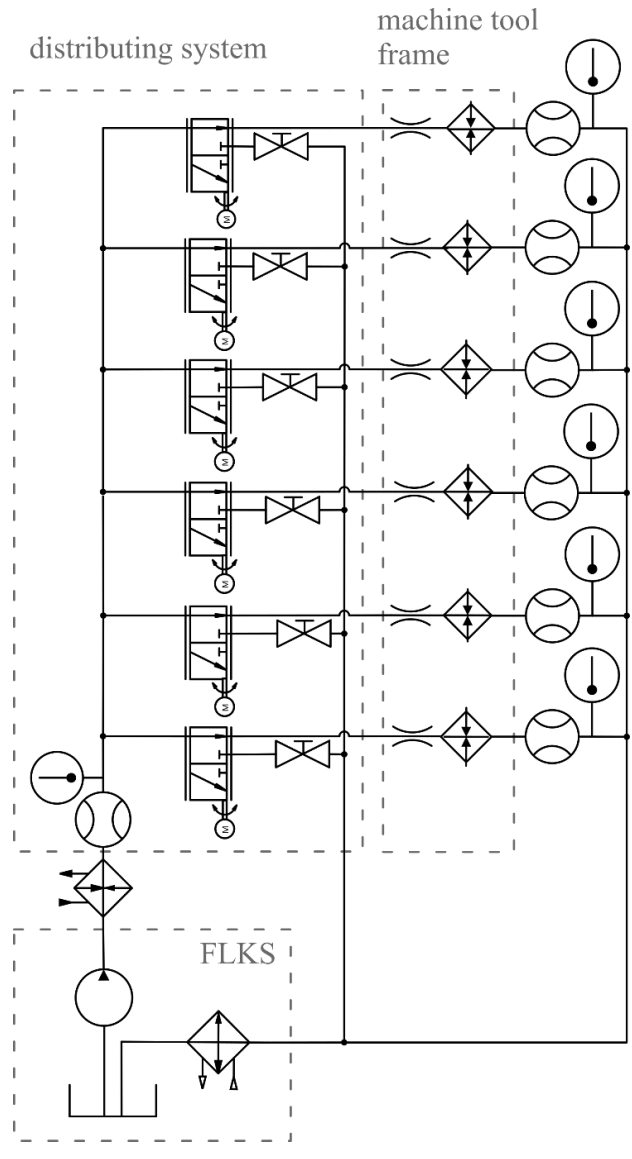

Figure 3: Schematic representation of the test rig

The sensors applied in the fluid system combine volume flow and temperature sensors. The volume flow sensors are based on the vortex principle [9]. One sensor is located in the common inlet and measures the total volume flow and additionally, each cooling circuit has one in the outlet for measuring the volume flow of each circuit. Figure 3 shows a block diagram of the test rig including the cooling system and the machine tool frame.

\section{SIMULATION MODEL}

The simulation model consists of two subsystems. The first is a network-based model of the cooling system. This model is a fast computable model, which is able to consider the fluid domain as well as the thermal domain. The second subsystem is a thermal FE simulation of the machine tool frame. To get a fast computable 
model one can use a MOR method. The MOR enables the user to obtain results within minutes instead of hours. The coupling between both systems is the wall temperature of the cooling channels inside the machine frame and the heat flux flowing through these walls. The cooling channels S1, S2, F1, and F2 are separated into 19 segments for spatial discretisation. The cooling channels M1 and M2 are so short that no discretisation is required. Overall, there are 78 segments for coupling.

\subsection{Network-based model of the cooling system}

The fluid circuit is simulated as a thermohydraulic network-based model with lumped parameters. The elements of the simulation model are e.g.:

- centrifugal pump for volume flow supply,

- volume elements with thermal and hydraulic ports,

- hydraulic resistances,

- variable throttle valves for setting the volume flow rate in each circuit and

- cooling pipes with heat exchange at the wall. The centrifugal pump has a volume flow-pressure characteristic stored and operates at a constant speed. The volume flow depends only on the pressure drop of the system. After the pump, a volume is placed. On its thermal port, heat is introduced into the system. This volume represents the pool heater. The amount of heat is temperature dependent. The control element is a two point controller with a hysteresis of $3.0 \mathrm{~K}$. The low level is $0 \mathrm{~W}$ and the high level is $3000 \mathrm{~W}$. There are seven branches after the heat input, the six cooling channels S1, S2, F1, F2, $\mathrm{M} 1$, and M2 as well as a bypass branch. The bypass has a constant flow resistance and leads back to the tank. An independently controlled variable throttle valve is placed at the beginning of each cooling channel. These valves regulate the flow resistance and determine the volume flow through each branch. Some additional hydraulic resistances and volumes represent the connecting hoses and fittings in the system. The cooling channels inside the machine tool frame are the key elements for heat exchange. A combination of hydraulic resistances and volumes with thermal ports is suitable for this purpose. The heat flow density $\dot{q}$, which is transferred from the fluid system to the machine frame, is calculated from the heat transfer coefficient (HTC) $\alpha$ and the temperature difference between the fluid temperature $T_{\mathrm{F}}$ and the wall temperature $T_{\mathrm{W}}$ :

$\dot{q}=\alpha \cdot\left(T_{\mathrm{F}}-T_{\mathrm{W}}\right)$.

The heat flow $\dot{Q}$ is calculated as follows:

$\dot{Q}=\dot{q} \cdot A$,

with the surface $A$ of the pipe segment. The HTCs are calculated in an a priori simulation and are parameters for the network-based model [10]. The FE machine frame model calculates the wall temperatures and the network-based model of the fluid system calculates the fluid temperatures itself.

\subsection{Modelling of the machine tool frame}

There are two models with distributed parameters for simulating the machine frame. First, a CFD model (computational fluid dynamics), which numerically calculates the temperature field and the HTCs of forced convection in the cooling channel within a conjugate heat transfer (CHT) simulation. Secondly, in the FE model, the temperature field of the machine frame can be calculated in simplified form using the parameters determined in the CFD. Both models are based on the computer aided design (CAD) model including the HPC machine frame and the integrated cooling pipes made of steel. The HTCs from the CFD simulation are also used in the network-based model of the cooling system, e. $g$ equation (1). They are calculated for a steadystate operating point. The authors know the dependency of the HTC on many variables, such as flow condition, temperature difference or the condition of the pipe surface. For the following investigations, they are initially assumed as constants. The reason for only using one steady state operating point is the high computational effort required by the CFD. To take the different workloads and environmental conditions into account, the surface of the machine tool, including the cooling pipes, is separated in different areas. Some of them perceive heat flows, the so-called Neumann boundary condition. For example, the 78 segments in which the cooling channels are separated and areas for working loads like power losses of drives and bearings. The second kind of boundary conditions are the Robin boundary conditions. For the environmental temperature load, three 
zones are defined. One zone is on the ground, which is the coldest point in most cases. The next one covers the lower part of the machine tool and the third zone is the top part. This separation is used due to the different air layers covering the machine tool frame. In order to reduce the computational effort and the time needed for the simulation, a MOR is performed on the FE model. The MOR is based on Krylov subspace methods. A description of the method can be found in [11].

It is possible to represent the thermal FE model as a multiple input multiple output (MIMO) state space model:

$$
\begin{aligned}
\dot{T}+A \cdot T & =B \cdot u \\
y & =C^{\mathrm{T}} \cdot T .
\end{aligned}
$$

The state is the temperature $T$ with its derivative $\dot{T}$. The input of the system is a vector $u$. This can be the ambient temperature or other loads on the machine frame. The system output $y$ returns the temperature at specified points of interest. $A, B$ and $C$ are matrices that describe the system with its inner interrelation as well as the in- and output behavior of the system. Physically, $A$ has elements describing conductivity and heat capacity. $B$ selects the nodes affected by thermal loads. Similar to $B$, matrix $C$ selects the output nodes. The dimension of $A$ in particular is very high, which explains the computational effort of the FE simulation. In the presented case, the degree of freedom (DoF) is 90854 . The size of matrix $A$ is $90854 \times 90854$. The reduced system looks structurally similar to the one in equation (3). It is given with

$$
\begin{aligned}
\dot{\hat{T}}+\hat{A} \cdot \hat{T} & =\hat{B} \cdot u \\
y & =\hat{C}^{\mathrm{T}} \cdot \hat{T} .
\end{aligned}
$$

The reduction is a projection into a Krylov subspace with a desired order. The projection rule is:

$\hat{A}=V^{\mathrm{T}} \cdot A \cdot V$

$\hat{B}=V^{\mathrm{T}} \cdot B$

$\hat{C}=V^{\mathrm{T}} \cdot C$

$T=V \cdot \hat{T}$.

The projection matrix $V$ is obtained with an Arnoldi algorithm [12, 13].

\subsection{Coupling of subsystems}

There are different types of software used. The meshing of the CFD and FE model is done in the ANSYS Workbench. An export of the mesh file

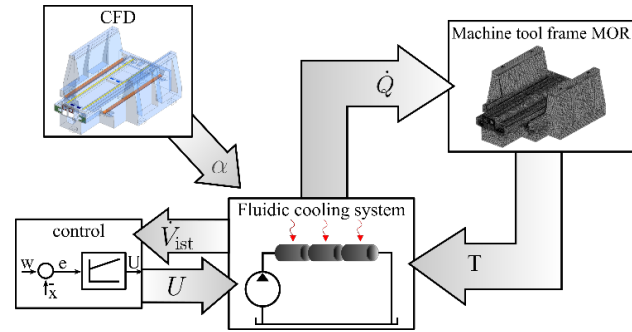

Figure 4: $\quad$ Coupling of the simulation subsystems

of the machine frame transfers data to MatLab, where one can perform the MOR. The software tool for implementing the network-based model of the cooling system is SimulationX. A C-code export gives offers the possibility to use the cooling system model in MatLab. With both subsystems, available in one software tool, the coupling is possible.

The coupling between the machine frame model and the model of the cooling system is done by exchanging thermal variables. The workflow is show in Figure 4. The heat flow density $\dot{q}$ is an output of the fluidic cooling system and input of the machine tool frame. In the other direction, the machine tool frames output is the pipe wall temperature $T$, which is an input for the fluid system. An external PI controller for each cooling channel enables the user to set a volume flow rate $\dot{V}$ for each channel.

\section{RESULTS}

To validate the coupled simulation model, five test cycles are used. The test cycles vary in the number of flooded cooling channels and the amount of volume flow through each circle. The settings for each test cycle are listed in Table 2. In test cases A and C, the machine tool frame is tempered symmetrically, whereas in the test cases $\mathrm{B}, \mathrm{D}$, and $\mathrm{E}$ an asymmetrical load is applied. Loads other than fluid and ambient temperature

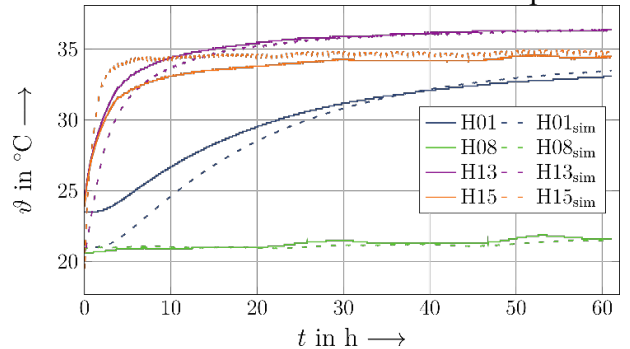

Figure 5: $\quad$ Test cycle A, sensors H1, H08, H13, H15, measurement and simulation 
Table 2

\begin{tabular}{|c|c|c|c|c|c|c|c|c|}
\hline \multirow{2}{*}{ 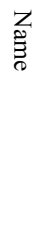 } & \multirow{2}{*}{ 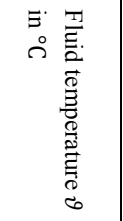 } & \multirow{2}{*}{ 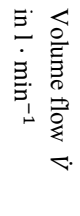 } & \multicolumn{6}{|c|}{ Cooling channel } \\
\hline & & & S1 & $\mathrm{F} 1$ & M1 & M2 & F2 & $\mathrm{S} 2$ \\
\hline A & $41.6-44.1$ & 4 & $\mathrm{x}$ & $\mathrm{x}$ & $\mathrm{x}$ & $\mathrm{x}$ & $\mathrm{x}$ & $\mathrm{x}$ \\
\hline B & $41.4-44.5$ & 4 & $\mathrm{x}$ & $\mathrm{x}$ & $\mathrm{x}$ & & & \\
\hline $\mathrm{C}$ & $40.5-42.9$ & 8 & $\mathrm{x}$ & $\mathrm{x}$ & $\mathrm{x}$ & $\mathrm{x}$ & $\mathrm{x}$ & $\mathrm{x}$ \\
\hline D & $41.4-44.0$ & 8 & $\mathrm{x}$ & $\mathrm{x}$ & $\mathrm{x}$ & & & \\
\hline $\mathrm{E}$ & $43.0-45.7$ & 15 & $\mathrm{x}$ & $\mathrm{x}$ & & & & \\
\hline
\end{tabular}

are not applied. For this reason, the cooling system works as a heater instead of a cooler. There was no possibility for installing heating pads or the like to emulate thermal loads properly. This is sufficient for validating the simulation. Each test cycle lasts almost three days. During this time, the temperature sensors in the machine frame and the sensors of the fluid system are recorded. Figure 5 shows the temperature chart for selected sensors for test cycle A. You can see that at sensor H08 that is located in the right side of the machine frame, far away from the fluid channels, only the ambient temperature has an influence. Here the temperature drift over three days is visible. A sensor near to the surface of the machine frame and near to a fluid channel is sensor H15. It is situated at channel M2, the motor cooling at the front of the machine frame. The ambient temperature and the fluid influence this sensor. It shows a rising of the temperature up to almost $35{ }^{\circ} \mathrm{C}$ with additional temperature fluctuations. Sensor H13 is close to the fluid pipe S2 and lies inside the machine frame. The ambient temperature has very little influence so the measured temperature rises more than the temperature at sensor $\mathrm{H} 15$. The last sensor shown in is sensor H01 located in the core of the machine frame. The heating through the fluid pipes only influences this sensor. Because of the distance between the sensor and the fluid channels the temperature rises not as strong as at sensors $\mathrm{H} 13$ and H15 that are nearer to the fluid pipes. After 62 hours you see that the machine frame is not in a thermal steady state. The temperature at sensor $\mathrm{H} 01$ rises slightly after this time. This shows the high thermal inertia of the machine frame.

At the beginning of the observed period, there is a difference between simulation and measurement. The simulation starts with a constant temperature for the entire solid frame. In Figure 5 you can see that the machine frame has different temperatures at the beginning of the measurement. Simulation and measurement get close at the end of the simulation.

Figure 6 shows the absolute error at the end of the simulation for the available sensors. A comparison of the different test cycles shows that the error behaviour of the five test cycles is similar. This discrepancy is due to different reasons. In the machine frame, reinforcing steel is installed from which the amount and position are not exactly known. The influence of the reinforcing steel is investigated in [14]. Therefore, there are unknown heat bridges in the machine frame that are not in the scope of the simulation. Another reason for the temperature

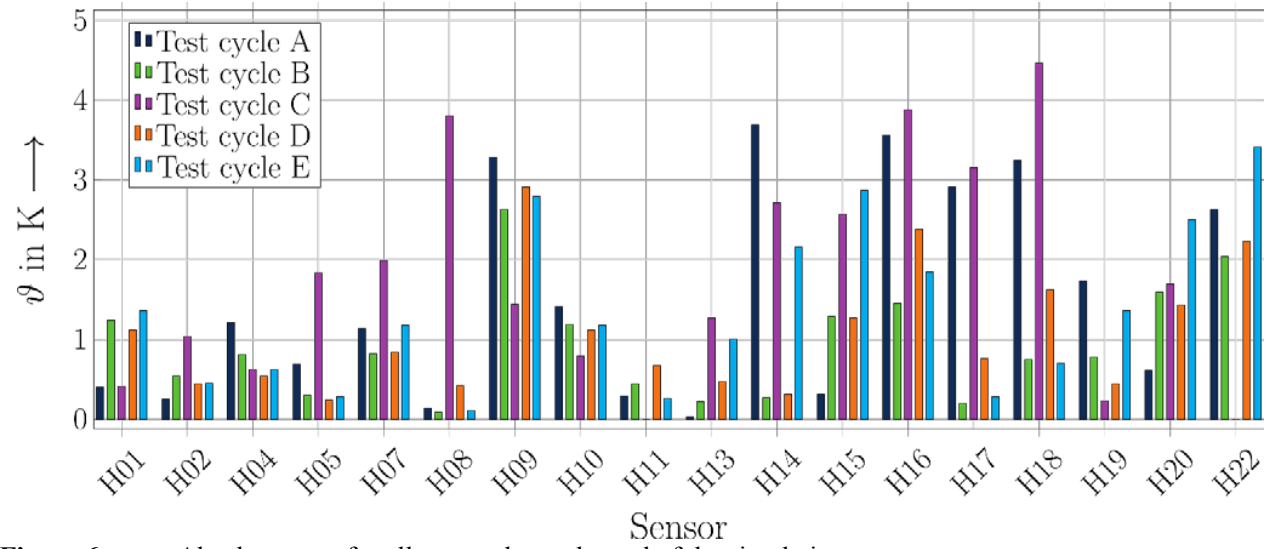

Figure 6: Absolute error for all test cycles at the end of the simulation 
difference is the constant starting temperature of the simulation.

\section{CONCLUSION AND OUTLOOK}

This paper presented a method for modelling a fast calculating thermal fluid co-simulation of a machine tool frame. The cooling system is modelled with lumped parameter methods and the machine frame is simulated with FE methods improved by MOR. The validation with six test cycles showed a good consistency between the measurements on the test rig and the simulation results.

Further investigation concerns an optimised machine tool frame with seven cooling channels. Four of these channels are placed in the sides of the U-shaped frame, two for the chip conveyer and one spreads through the floor area. Furthermore, the cooling system has to be adapted. Small centrifugal pumps are used for each channel to improve the controllability of the volume flow. Additionally, the number of cooling circuits is fitted to the number of channels in the machine frame. These measures are intended to improve the thermal stability of the machine tool frame in terms of time and location.

\section{NOMENCLATURE}

$\begin{array}{lll}A & \text { Area } & \mathrm{m}^{2} \\ A & \text { System matrix } & \text { div. } \\ B & \text { Input matrix } & \text { div. } \\ C & \text { Output matrix } & \text { div. } \\ \dot{Q} & \text { Heat flow } & \mathrm{W} \\ T & \text { Temperature } & \mathrm{K} \\ V & \text { Projection matrix } & - \\ \dot{V} & \text { Volume flow } & \mathrm{m}^{3} \cdot \mathrm{s}^{-1} \\ \dot{q} & \text { Heat flow density } & \mathrm{W} \cdot \mathrm{m}^{-2} \\ u & \text { Input state space model } & \mathrm{div} \cdot \\ \mathrm{y} & \text { Output state space model } & \mathrm{div} . \\ \alpha & \text { Heat transfer coefficient } & \mathrm{W} \cdot \mathrm{m}^{-2} \cdot \mathrm{K}^{-1} \\ \vartheta & \text { Temperature } & \mathrm{K},{ }^{\circ} \mathrm{C} \\ C A D & \text { Computer aided design } & \\ C F D & \text { Computational fluid dynamics } & \\ C H T & \text { Conjugate heat transfer } & \\ D o F & \text { Degree of Freedom } & \\ F E & \text { Finite element } & \\ F E M & \text { Finite element method } & \\ F L K S & \text { fluid/air cooling system } & \\ H P C & \text { High performance concrete } & \\ H T C & \text { Heat transfer coefficient } & \\ M I M O & \text { Multiple input multiple output } & \\ M O R & \text { model order reduction } & \\ T C P & \text { tool centre point } & \\ & & \end{array}$

\section{ACKNOWLEDGEMENT}

The presented research activities are part of the transfer project T02 "Robust and thermo-energetically optimal tempering of machine tool frames with fluidic tempering systems funded by the German Research Foundation - Project-ID 174223256 - TRR 96.

The authors would like to thank the German Research Foundation (DFG) and all project partners including HYDAC Cooling $\mathrm{GmbH}$, FRAMAG Industrieanlagenbau $\mathrm{GmbH}$ and METROM Mechatronische Werkzeugmaschinen $\mathrm{GmbH}$ for the financial support.

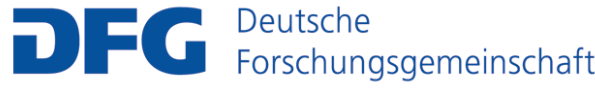




\section{REFERENCES}

[1] Brecher C (ed.) (2012) Effizienzsteigerung von Werkzeugmaschinen durch Optimierung der Technologien zum Komponentenbetrieb EWOTeK, 1. Aufl. Apprimus, Aachen

[2] Denkena B (2015) Leanergie:

Lebenszyklusorientierte Lösungen und Dienstleistungen für Gestaltung und Betrieb energieeffizienter Werkzeugmaschinen : Abschlussbericht Leibniz Universität Hannover, Institut für Fertigungstechnik und Werkzeugmaschinen

[3] Denkena B, Garber T (eds) (2013) NC Plus: Prozess- und wertschöpfungsorientiert gesteuerte Werkzeugmaschine; Abschlussbericht. Berichte aus dem IFW. PZH-Verl., Garbsen

[4] Mayr J, Jedrzejewski J, Uhlmann E et al. (2012) Thermal issues in machine tools. CIRP Annals 61(2): 771-791.

[5] Shabi L, Weber J, Weber J (2018) Simulative Study of Different Control Concepts of Cooling System for Machine Tools. MATEC Web Conf.

[6] Shabi L, Weber J, Weber J (2016) Investigation of fluidic systems in machine tools. International Conference on Hydraulics and Pneumatics HERVEX

[7] Lohmann B, Salimbahrami B (2004)

Ordnungsreduktion mittels Krylov-

Unterraummethoden (Order Reduction using Krylov Subspace Methods). at -

Automatisierungstechnik 52(1-2004): 30-38.

[8] Beitelschmidt M, Galant A, Großmann K et al. (2015) Innovative Simulation Technology for Real-Time Calculation of the Thermo-Elastic Behaviour of Machine Tools in Motion. AMM 794: 363-370.

[9] Richardson DA, Robinson RJ (1974) Vortex flow meter transmitter including piezo-electric sensors (US3948098A)

[10] Weber J, Glänzel J, Popken J et al. (2019) Combined and fast computable thermal models for situationally optimal tempering of machine tool components. Procedia CIRP 79: 608-613.

[11] Galant A, Beitelschmidt M, Großmann K (2016) Fast High-Resolution FE-based Simulation of Thermo-Elastic Behaviour of Machine Tool Structures. Procedia CIRP 46: 627-630.

[12] Arnoldi WE (1951) The principle of minimized iterations in the solution of the matrix eigenvalue problem. Quart. Appl. Math. 9(1): 17-29.

[13] Salimbahrami B, Lohmann B (2002) Krylov subspace methods in linear model order reduchtion: Introduction and invariance properties

[14] Glänzel J, Geist A, Ihlenfeldt S (2019) Simulation-based investigation for heat transfer behavior of steel reinforcements in concrete machine frames and their thermal effects. Journal of Machine Engineering Vol. 19 No 3 17-30 